\title{
Das SGUM-Zertifikat Notfallsonographie
}

\section{Joseph Osterwalder}

Leiter Arbeitsgruppe Notfallsonographie Sektion Allgemeine Innere Medizin/SGUM sowie Sprecher Arbeitskreis Notfallsonographie DEGUM

* Die Literaturangaben finden sich im Internet unter www.saez.ch $\rightarrow$ Aktuelle Nummer oder $\rightarrow$ Archiv $\rightarrow 2012 \rightarrow 34$.
Korrespondenz:

PD Dr. Joseph Osterwalder MPH Zentrale Notfallaufnahme

Kantonsspital

CH-9007 St. Gallen

joseph.osterwalder[at]kssg.ch
Ein Bild ist mehr wert als 1000 Worte.

\section{Ausgangslage}

Bis Mitte der 90er Jahre galt in der Sonographie ein eisernes Prinzip: Die einzelne Untersuchung durfte nur durch klardefinierte, hochqualifizierte Spezialisten erfolgen und musste immer eine ganze Region (z.B. Abdomen), eine physiologische Einheit (z.B. Urogenitalapparat) oder ein Organ (z. B. Herz) umfassen. Weiter galt es zwingend, alle möglichen, sonographisch in Frage kommenden Pathologien zu überprüfen. Mit der Einführung des nordamerikanischen «FAST» (Focused Assessment with Sonography for Trauma), begann das Dogma der formalen Sonographie zu bröckeln. Dabei handelte es sich nur um eine Wiederentdeckung der Vorgehensweise deutscher Chirurgen aus den 80er-Jahren. Im Zentrum stand die Idee einer Übersetzung klinischer Probleme in einfache sonographische Ja-/Nein-Fragestellungen. Bei der FAST-Untersuchung ging es um den Nachweis von Flüssigkeit - im Traumakonzept gleichbedeutend mit Blut - in der Peritoneal-, Pleura- und Perikardhöhle. Seither kamen zahlreiche weitere Ja-/NeinFragestellungen im Rahmen unterschiedlichster Anwendungen für die Notfallmedizin dazu. 2009 hat das ACEP (American College of Emergency Physicians) einen entsprechenden, wissenschaftlich abgestützten Indikationenkatalog publiziert [1]*. Auf dieser Grundlage stellte eine Arbeitsgruppe renommierter Ultraschallspezialisten der deutschen, schweizerischen und österreichischen Gesellschaft für Ultraschall in der Medizin (s. Tab.) ein länderübergreifendes Konzept zur Basisausbildung in Notfallsonographie (NFS) zusammen. Im Oktober 2010 wurde das Programm von den 3 entsprechenden Vorständen verabschiedet [2]. Nachfolgend stellen wir das Konzept und dessen Umsetzung in der Schweiz vor.

\begin{tabular}{ll}
$\begin{array}{l}\text { 3-Länder-Arbeitsgruppe Basisausbildung Notfallsonographie } \\
\text { Fachbereich }\end{array}$ & Personen/Ultraschallgesellschaft \\
\hline Anästhesie/Intensivmedizin & PD Dr. Raoul Breitkreutz (DEGUM)* \\
\hline Allgemeine Medizin & Dr. Kurt Somavilla (ÖGUM)* \\
\hline Innere Medizin/ & PD Dr. Karl-Heinz Seitz (DEGUM) \\
Gastroenterologie & Dr. Wolfgang Blank (DEGUM) \\
& Prof. Dr. Gebhard Mathis (ÖGUM) \\
\hline Kardiologie & Prof. Dr. Andreas Hagendorf (DEGUM) \\
& Prof Dr. Thomas Binder (ÖGUM) \\
Notfallmedizin & Dr. Barbara Hogan (DEGUM) \\
& Dr. Rudolf Horn (SGUM)* \\
& PD Dr. Joseph Osterwalder (SGUM)
\end{tabular}

* DEGUM: Deutsche Gesellschaft für Ultraschall in der Medizin ÖGUM: Österreichische Gesellschaft für Ultraschall in der Medizin SGUM: Schweizerische Gesellschaft für Ultraschall in der Medizin

\section{Definition und Begründung}

Unter NFS verstehen wir die Anwendung der Ultraschalltechnologie am Patientenbett:

- im Rahmen einer organ- und fächerübergreifenden Evaluation sowie Behandlung von Notfallpatienten

- bei schwierigen oder risikoreichen invasiven Verfahren

- für das Monitoring von ausgewählten Vitalparametern [3].

Die Durchführung, Interpretation sowie Integration in die Gesamtbeurteilung wird nicht an einen Spezialisten delegiert, sondern ist Sache des behandelnden Arztes [3]. Die aus der fokussierten Sonographie resultierenden Zusatzinformationen sollen den in Ausbildung stehenden Ärzten sowie Generalisten helfen, lebensbedrohliche oder häufige Zustände schneller und besser zu erkennen und zu behandeln sowie ohne Verzögerung Spezialistenhilfe anzufordern. Allerdings kann die NFS keineswegs die formale Untersuchung durch den Spezialisten ersetzen. Sie erlaubt jedoch, Entscheidungen, welche mit oder ohne Sonographie anstehen, in kürzerer Zeit und mit grösserer Sicherheit zu fällen. Eine Vielzahl von Publikationen belegt diese Aussage [4-13].

\section{Wer soll in NFS ausgebildet werden?}

In schweizerischen Spitälern erfolgt die Erstbeurteilung und Erstbehandlung von Notfallpatienten in der Regel durch Ärzte in der Weiterbildung und nicht durch Fachspezialisten. Aus einer Umfrage der SGNOR [14] wissen wir, dass $64 \%$ der Spitäler keine vorgängige praktische Erfahrung für den ärztlichen Notfalldienst fordern, 32\% mindestens 6 Monate und nur $4 \% 2$ Jahre oder mehr. Dabei sind die Spezialisten im Hintergrund 24 Stunden/7 Tage abrufbereit (Internisten und Chirurgen in 97\%, Anästhesisten in 94\%, Radiologen in $86 \%$ und Kardiologen in 57\%). Allerdings sind die Fachärzte nur in 74\%, resp. 66\%, 89\%, $55 \%$ und $53 \%$ innerhalb von 15 Minuten vor Ort. So müssen mehrheitlich unerfahrene Ärzte erste wichtige und schwierige Entscheidungen fällen. Dabei hilft ihnen die NFS, weil die Basisapplikationen einfach und schnell erlernbar sind sowie problemlos in die klinische Untersuchung integriert werden können.

\section{Weiterbildung in Notfallsonographie}

Die Weiterbildung in NFS soll möglichst vielen jungen Kollegen einen qualitativ hochstehenden Einstieg in die Sonographie erleichtern und ermöglichen. Gleichzeitig wird mit vertretbarem Aufwand ein grösstmöglicher Nutzen für die Notfallversorgung angestrebt. Die angebotene Weiterbildung führt zum Zertifikat Notfallsonographie und verfolgt 7 Ziele [2]: 
1. Korrekte Anlotung/Bildgebung und allgemeine Bild- sowie Befundinterpretation

2. Basiskenntnisse und -fertigkeiten der Sonographie, die im Grundkurs Abdomen vermittelt werden

3. Integration der Sonographie in den Untersuchungs- und Behandlungsablauf

4. E-FAST: Suche nach Hämoperitoneum, Hämoperikard, Hämothorax und Pneumothorax

5. Fokussierte Sonographie mit folgenden Fragen: Abdominales Aortenaneurysma (AAA), Gallensteine, Blasenstatus und Nierenaufstau, Tiefe Venenthrombosen in der Inguina sowie Poplitea, Struktur und Funktion des Herzens, d. h. Herzdimensionen, globale systolische Funktion, Rechtsherzbelastung, Perikarderguss und dynamischer Volumenstatus

6. Ultraschallgestützte Punktionen (Gefässe, Aszites und Pleuraerguss

7. Dokumentation.

\section{Interimsreglement für die Weiterbildung zum Zertifikat Notfallsonographie [15]}

Details dazu findet man unter www.sgum.ch $\rightarrow$ AG Notfallsonographie.

Im Interimsreglement werden die Weiterbildung

für das Zertifikat Notfallsonographie geregelt sowie die Struktur und Organisation für eine erste Aufbauphase festgelegt. Die Struktur zeigt Abbildung 1.

Die Weiterbildung wurde im 3-Länder-Curriculum definiert [2]. Sie besteht aus 2 Kursen [Basisnotfallsonographie (BNFS) und fokussierte Echokardiographie (FECHO)] sowie 200 supervidierten Untersuchungen (120 BNFS und 80 FECHO). Für den Kurs BNFS existieren 2 Formate: Format 1: Bestehender 21/2-tägiger Grundkurs Abdomen und 1-tägiger Grundkurs NFS. Falls der Grundkurs NFS vor dem

\section{Zertifikat Notfallsonographie}

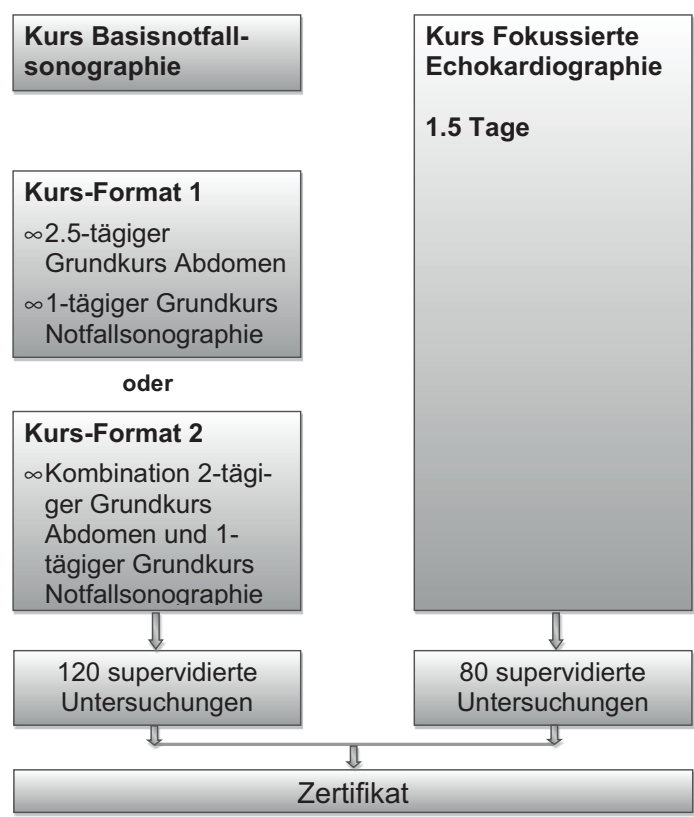

Grundkurs Abdomen besucht wird, sind im Grundkurs NFS 90 Minuten für die technischen Grundlagen M-Mode einzuplanen (30 Minuten Theorie, 1 Stunde praktische Übung, Gesamtkursdauer 9,5 Stunden); Format 2: Kombination eines verkürzten, 2-tägigen Grundkurses Abdomen mit einem 1-tägigen Grundkurs NFS. Die Dauer für den Kurs FECHO beträgt mind. 12 Std. Die Anwesenheit eines Kardiologen im Tutorenteam ist obligatorisch.

\section{Praxis}

Es werden 200 supervidierte Untersuchungen für das Zertifikat gefordert:

- 25 E-FAST-Untersuchungen (mind. 5 pathologisch)

- 20 AAA-Untersuchungen (mind. 5 pathologisch)

- 20 Gallenblasenuntersuchungen (mind. 5 pathologisch)

- 20 Blasenstatus- und Nierenaufstauuntersuchungen (mind. 5 pathologisch)

- 25 Venenuntersuchungen (mind. 5 pathologisch)

- 80 fokussierte Echokardiographien (mind. 20 mit einer der 4 geforderten Pathologien)

\section{Zertifikat NFS}

Das Zertifikat NFS (Abb.) stellt eine Zwischenstufe zu einem geplanten Fähigkeitsausweis (FA) Notfallsonographie dar, soll aber auch für den FA oder weitere in Frage kommende Fähigkeitsausweise gelten. Es ist nicht tarifwirksam, sondern soll jungen Kollegen einen niederschwelligen, qualitativ hochstehenden Einstieg in die Sonographie erlauben, die Routineversorgung in Notfallstationen verbessern und zur Weiterbildung zum FA motivieren.

\section{Organisation}

Das Zertifikat NFS wird von einer Arbeitsgruppe NFS in der Sektion Allgemeine Innere Medizin verwaltet. Anfragen zur Anerkennung von Kursen, Supervisionen und zur Berechtigung für Tutor oder Kursleiter sowie zur Supervision sind an die Weiterbildungskommission der Sektion Allgemeine Innere Medizin zu richten. Für Anträge zum Zertifikat NFS ist ebenfalls die Weiterbildungskommission zuständig. Diese Dienstleistungen sind gemäss SGUM-Standard gebührenpflichtig.

\section{Ausblick}

Anlässlich des 3-Länder-Treffens im September 2012 in Davos wird eine neue Sektion CAN (Chirurgen, Anästhesisten, Notfallmediziner) gegründet. Die Belange der Notfallsonographie bleiben unter der Führung der Arbeitsgruppe NFS, bis sich die neue Sektion etabliert hat. Die neue Sektion arbeitet jedoch an einem Fähigkeitsausweis für die 3 Fachbereiche, welcher modular erworben werden kann.

Weitere Auskünfte und Anlaufstelle: Sekretariat SGUM, Frau A. Benyamini, Tel. 04482511 33, sgum[at]hin.ch 